\title{
NHÂN XÉT KẾT QUẢ CHỌC HÚT TẾ BÀO KIM NHỎ VÀ SINH THIỂT TỨC THİ TRONG CHẨN ĐOÁN UNG THƯ TUYẾN GIÁP
}

\section{TÓM TẮT}

Mục tiêu: Nhận xét chỉ định và kết quả chẩn đoán ung thư tuyến giáp bằng xét nghiệm chọc hút kim nhỏ tế bào học và sinh thiết tức thì tại Bệnh viện Quân y 103. Đối tượng và phương pháp: Nghiên cứu hồi cứu, trên nhửng bệnh nhân được chẩn đoán xác định ung thư tuyến giáp theo kết quả mô bênh học sau mổ. Đối chiếu kết quả tế bào học và mô bệnh tức thì với kết quả mô bệnh sau mổ. Kết quả: 106 bệnh nhân ung thư tuyến giáp được phấu thuật cắt toàn bộ tuyến giáp. Xét nghiệm tế bào bằng chọc hút kim nhỏ trước mổ được thực hiện cho $100 \%$ trường hợp, kết quả: xác đinh được 51 bệnh nhân là ung thư (tỷ lệ 48,1\%) và 50 bệnh nhân (tỷ lệ 42,7\%) nghi ngờ ung thư tuyến giáp; 4 trường hợp chẩn đoán lành tính. STTT được chỉ định khi kết quả FNA (tỷ lệ 90,9\%) hoặc khi tổn thương được đánh giá đại thể trong mổ (tỷ lệ $9,1 \%)$ nghi ngờ ác tính. Kết quả định típ tế bào của xét nghiệm STTT phù hợp với giải phấu bệnh đạt 85,5\%; STTT không xác định được các biến thể nang và tế bào trụ của UTBMTG thể nhú (tỷ lệ 14,5\%). Kêt Iuận: Hiệu quả chẩn đoán UTBMTG bằng chọc hút tế bào kim nhỏ đạt 48,1\%. Cần chỉ định STTT cho những trường hợp có kết quả FNA nghi ngờ UTBMTG và những trường hợp ghi nhận tổn thương đại thể bất thường trong mổ để nâng cao kết quả chẩn đoán.

Tư khóa: Ung thư tuyến giáp, chọc hút kim nhỏ tế bào học, sinh thiết tức thì

\section{SUMMARY}

\section{COMMENT ON THE RESULTS OF THE} APPLICATION OF FINE -NEEDLE ASPIRATION AND FROZEN SECTION IN DIAGNOSING OF THYROID CARCINOMA

Objective: To review indications and diagnostic results of thyroid cancer by fine-needle aspiration (FNA) and frozen section at Military Hospital 103. Subjects and methods: Retrospective study on patients were diagnosed with thyroid cancer according to histopathological results. Compare the cytological and immediately histopathological results with the HE stain results. Results: 106 thyroid cancer patients underwent total thyroidectomy. Preoperative fine needle aspiration cytology was performed for $100 \%$ of cases, the results: identified of 51 patients with cancer (rate 48.1\%) and 50 patients (rate 42.7) \%) suspected thyroid cancer; 4 cases were diagnosed as benign. Frozen-section was mainly indicated for patients with suspected of malignancy by FNA results

*Bệnh viện 103, Hoc viện Quân Y

Chịu trách nhiệm chính: Vũ Anh Hải

Email: vuanhhai.ncs@gmail.com;

Ngày nhận bài: $1 / 8 / 2021$

Ngày phản biện khoa học: 30/8/2021

Ngày duyệt bài: 20/9/2021

\section{Vũ Anh Hải*, Lê Việt Anh*, Trần Xuân Bộ*}

$(90.9 \%)$ or macroscopic lesion during operation $(9,1 \%)$. The histopathologic type identification of frozen section test were consistent with $\mathrm{HE}$ stain at rate of $85.5 \%$; frozen section test did not identify follicular and columnar variants of papillary thyroid carcinoma (14.5\%). Conclusions: The efficiency of thyroid cancer diagnosis by FNA was $48.1 \%$. Frozen section should be indicated for cases with FNA results or macroscopic lesions suspected of thyroid cancer to improve diagnostic results.

Key words: Thyroid cancer, fine-needle aspiration (FNA), frozen section.

\section{I. ĐẶT VẤN ĐỀ}

Ung thư biểu mô tuyến giáp (UTBMTG) là bệnh ác tính thường gặp, chiếm $90 \%$ các trường hợp ung thư tuyến nội tiết và khoảng $1 \%$ các loại ung thư [1], [2]. Tại Việt Nam, theo số liệu Globocan, số ca mắc mới trong năm 2020 là 5471 trường hợp, chiếm tỉ lệ $3 \%$ và đứng thứ 10 trong tổng số các ca mắc ung thư, số ca tử vong là $642 \mathrm{ca}$, chiếm tỉ lệ 0,52\% [3].

Chẩn đoán xác định bản chất bướu giáp trước phẫu thuật rất quan trọng, từ đó lựa chọn chiến lược điều tri phù hợp. Chọc hút tế bào bằng kim nhỏ (FNA) là xét nghiệm an toàn, dễ thực hiện, được áp dụng phổ biến trong chẩn đoán. Tuy vậy, độ nhạy, độ đặc hiệu vẩn còn những hạn chế. Do vậy, trong nhiều trường hợp, xét nghiệm mô học tức thì trong mổ (STTT) cân được thực hiện để chẩn đoán bản chất khối u [4], [5].

Trong nghiên cứu này, chúng tôi thực hiện hồi cứu trên 106 bênh nhân đã được chẩn đoán bằng FNA trước phẩu thuật hoặc STTT trong mổ. Từ đó, rút ra một số nhận xét về chỉ định và kết quả của hai xét nghiệm này tại Bệnh viện Quân Y 103.

\section{II. ĐỐI TƯỢNG VÀ PHƯƠNG PHÁP NGHIÊN CỨU}

2.1. Đối tượng. 106 bệnh nhân UTBMTG được phẫu thuật cắt hoàn toàn tuyến giáp tại khoa Phẫu thuật Lồng ngực - Bệnh viện Quân y 103 , từ tháng 6/2018 đến 12/2020.

2.2. Phương pháp. Nghiên cứu hồi cứu, mô tả cắt ngang.

\subsection{Chỉ tiêu nghiên cứu}

- Đặc điểm nhóm bệnh nhân nghiên cứu: Tuổi, giới tính, kích thước khối u, phân nhóm Tirads, giai đoạn TNM $8^{\text {th }}$.

- Nhận xét chỉ định và đánh giá kết quả của FNA và STTT: chỉ định theo kích thước u, phân nhóm 
Tirads; kết quả chẩn đoán và định típ mô bênh.

2.4. Xử lý số liệu. Số liệu được thu thập theo mẫu bệnh án thống nhất, xử lý các thuật toán bằng phần mềm SPSS 24.0.

\section{KẾT QUẢ NGHIÊN CỨU}

3.1. Đặc điểm bệnh nhân nghiên cứu

Bảng 1. Đặc điểm bệnh nhân nghiên cứu

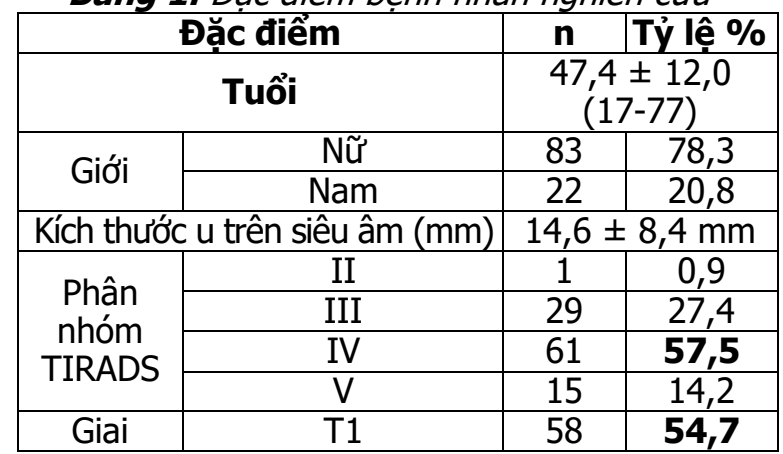

\begin{tabular}{|c|c|c|c|}
\hline \multirow[t]{3}{*}{ đoạn pT } & T2 & 4 & 3,8 \\
\hline & T3 & 26 & 24,5 \\
\hline & T4 & 18 & 17,0 \\
\hline \multirow{4}{*}{$\begin{array}{c}\text { Giai } \\
\text { đoạn } \\
\text { pTNM }\end{array}$} & I & 90 & 84,9 \\
\hline & II & 11 & 10,4 \\
\hline & III & 4 & 3,8 \\
\hline & IV & 1 & 0,9 \\
\hline \multirow{2}{*}{$\begin{array}{c}\text { Di căn } \\
\text { hạch cổ }\end{array}$} & Có & 51 & 48,1 \\
\hline & Không & 55 & 51,9 \\
\hline \multirow{3}{*}{$\begin{array}{l}\text { Mô } \\
\text { bệnh } \\
\text { học }\end{array}$} & UTBMTG thế nhú & 88 & 83,0 \\
\hline & $\begin{array}{c}\text { Biễn thế của } \\
\text { UTBMTG thể nhú }\end{array}$ & 18 & 17,0 \\
\hline & UTBMTG thế nang & 0 & \\
\hline
\end{tabular}

Tỉ lệ nữ:nam là 4:1. Đánh giá bướu giáp trên siêu âm chủ yếu thuộc phân nhóm TIRADS IV (57,5\%). Giai đoạn T1 chiếm tỷ lệ cao (54,7\%). UTBMTG biệt hóa ở giai đoạn I chiếm chủ yếu (tỷ lệ 83,3\%).

\subsection{Kêt quả ứng dụng FNA}

Bảng 2. Chỉ định FNA theo kích thước u và phân nhóm Tirads

\begin{tabular}{|c|c|c|c|c|c|}
\hline \multirow{2}{*}{ Kích thước u } & \multicolumn{4}{|c|}{ Tirads } & \multirow{2}{*}{ Tổng } \\
\cline { 2 - 6 } & II & III & IV & V & 40 \\
\hline$<10$ & 1 & 11 & 25 & 3 & 47 \\
\hline $10-<20$ & 0 & 12 & 26 & 9 & 19 \\
\hline$\geq 20$ & 0 & 6 & 10 & 3 & 106 \\
\hline Tống & 1 & 29 & 61 & 15 & .
\end{tabular}

Xét nghiệm FNA được chỉ định khi khối u kích thước dưới $10 \mathrm{~mm}$ chiếm tỷ lệ $37,7 \%$, trong đó chủ yếu thuộc phân nhóm từ TIRADS III trở lên (39/40 trường hợp), phân nhóm TIRADS II chiếm tỷ lệ rất thấp $(1 / 40$ trường hợp $=2,5 \%)$.

Bảng 3. Kết quả chẩn đoán tế bào học

\begin{tabular}{|c|c|c|}
\hline Tế bào học & \multicolumn{2}{|c|}{ Tống (n = 106) } \\
\cline { 2 - 3 } Ung thư & $\mathbf{n}$ & $\mathbf{\%}$ \\
\hline Nghi ngờ ung thư & 51 & 48,1 \\
\hline Lành tính & 50 & 47,2 \\
\hline
\end{tabular}

FNA có độ nhạy cao trong chấn đoán UTBMTG (tỷ lệ 95,3\%).

Bảng 4. Kết quả FNA theo kích thước u

\begin{tabular}{|c|c|c|c|c|}
\hline $\begin{array}{c}\text { Kích thước u } \\
(\mathbf{m m})\end{array}$ & \multicolumn{3}{|c|}{ FNA } & \multirow{2}{*}{ Tổng } \\
\cline { 2 - 4 } & Ung thư & Nghi ngờ & Lành tính & 40 \\
\hline$<\mathbf{1 0}$ & 16 & 22 & $\mathbf{2}$ & 47 \\
\hline $\mathbf{1 0}-\mathbf{2 0}$ & 24 & 20 & $\mathbf{3}$ & 19 \\
\hline$>\mathbf{2 0}$ & 11 & 8 & $\mathbf{0}$ & 106 \\
\hline Tống & 51 & 50 & $\mathbf{5}$ & \\
\hline
\end{tabular}

FNA có độ nhạy cao trong chấn đoán UTBMTG với u kích thước lớn, trên 20mm (19/19 trường hợp, tỷ lệ 100\%).

3.3. Kết quả ứng dụng STTT

Bảng 5. Chi định STTT theo kêt quả FNA và phân nhóm Tirads

\begin{tabular}{|c|c|c|c|c|c|}
\hline \multirow{2}{*}{ Tế bào học } & \multicolumn{3}{|c|}{ Tirads } & \multirow{2}{*}{ Tổng } \\
\cline { 2 - 5 } & II & III & IV & V & \\
\hline Lành tính & 0 & 4 & 0 & 1 & 5 \\
\hline Nghi ngờ ung thư & 1 & 17 & 28 & 4 & 50 \\
\hline Tống & $\mathbf{1}$ & $\mathbf{2 1}$ & $\mathbf{2 8}$ & $\mathbf{5}$ & $\mathbf{5 5}$ \\
\hline
\end{tabular}

Tất cả bệnh nhân có kết quả FNA nghi ngờ ác tính đè được làm STTT để xác định chẩn đoán trước khi quyết định cắt toàn bộ tuyến giáp. 4 trường hợp có kểt quả FNA lành tính, tirads III nhưng đánh giá đại thể trong mổ, tổn thương có dấu hiệu xâm lấn, do vậy được thực hiện STIT để chẩn đoán. 
Bảng 6. So sánh kêt quả định típ tế bào giữa STTT với giải phẫu bệnh

\begin{tabular}{|c|c|c|c|c|}
\hline \multirow[b]{2}{*}{ STT } & \multicolumn{3}{|c|}{ Mô bệnh học } & \multirow[t]{2}{*}{ Tống } \\
\hline & $\begin{array}{c}\text { Carcinoma } \\
\text { thể nhú }\end{array}$ & $\begin{array}{l}\text { Carcinoma nhú } \\
\text { biến thể nang }\end{array}$ & $\begin{array}{l}\text { Carcinoma nhú } \\
\text { biến thể tế bào trụ }\end{array}$ & \\
\hline Carcinoma thế nhú & 47 & 7 & 1 & 55 \\
\hline Biến thế PCT & 0 & 0 & 0 & 0 \\
\hline Tống & 47 & 7 & 1 & 55 \\
\hline
\end{tabular}

Tỷ lệ phù hợp trong định típ mô học là 85,5\%; xét nghiệm mô học tức thì không xác định được các biến thể của ung thư tuyến giáp thể nhú (0/8 trường hợp).

\section{BÀN LUẦN}

Ung thư biểu mô tuyến giáp thể nhú là bệnh lí ác tính phổ biến nhất của tuyến giáp, khả nằng bệnh nhân được chẩn đoán chính xác cao khi được chỉ định FNA các tổn thương tuyến giáp khi kết quả siêu âm có nghi ngờ. FNA được coi là xét nghiệm đáng tin cậy, tiết kiệm chi phí và ít xâm lấn nhất để chẩn đoán nhân tuyến giáp. Tuy nhiên, trong một số trường hợp kết quả FNA không chính xác, do những nguyên nhân như: kĩ thuật lấy bệnh phẩm không đây đủ, kinh nghiệm của bác sĩ giải phẫu bệnh và các đặc điểm tế bào học trùng lặp giữa u nang lành tính và ác tính [5].

STTT trong mổ là công cụ chẩn đoán được đánh giá là có độ chính xác cao, hạn chế được những nguyên nhân gây sai lệch kết quả của FNA do khả năng lấy được chính các tổn thương và kích thước mẫu bệnh phẩm lớn... Kết quả STTT là cơ sở, giúp phẩu thuật viện đưa ra quyết định xử trí tốn thương ngay trong cuộc mổ. Do đó, quan điểm áp dụng STTT trong các trường hợp chẩn đoán FNA nghi ngờ ác tínhc được đa số phẩu thuật viên đồng thuận, thực hiện [6], [7].

Nhiều nghiên cứu cho thấy độ đặc hiệu của FNA đối với ung thư tuyến giáp (không bao gồm các trường hợp FNA nghi ngờ) trên $90 \%$, từ đó cho rằng STTiT sẽ không hữu ích [5], [7], [8]. Qin Y., và cs (2017), nghiên cứu trên 1265 bệnh nhân được thực hiện FNA và STTT, kết quả: nhóm bệnh nhẩn FNA ác tính có $10,0 \%$ STTT là lành tính hoặc không xác định, trong số đó $96,4 \%$ bệnh nhân có giải phẩu bệnh là ác tính [5]. Một số tác giả cũng khuyến cáo, kết quả STTT có tỷ lệ âm tính giả khá cao. Theo Kenedy J.M., và cs (2016), Qin Y., và cs (2017), thống kê các trường hợp có kết quả STTT là lành tính thì có tới $50 \%$ đển $80 \%$ trong số đó có kết quả giải phẫu bệnh là ác tính [5]. Như vậy, STTT không phải là xét nghiệm có độ chính xác cao trong loại trừ bệnh lý ác tính.

Nghiên cứu của chúng tôi cho thấy, xét nghiệm FNA cho kết quả nghi ngờ ác tính chiếm tì lệ khá lớn $(47,2 \%)$; tuy vậy nếu xét về độ nhạy của FNA trong chẩn đoán ung thư tuyến giáp, tỷ lệ lên tới 95,3\%. Như vậy, FNA có hiệu quả cao trong chẩn đoán cao, việc đưa ra kết quả "nghi ngờ" là do các nhà tế bào học tại cơ cở chúng tôi đã thận trọng hơn trong việc đưa ra chẩn đoán xác định là ác tính. Kết quả nghiên cứu của chúng tôi cũng đã chứng minh STTT là rất hữu ích trong việc xác chẩn bệnh ung thư tuyến giáp, với $100 \%$ trường hợp có kết quả FNA nghi ngờ được xác chẩn chính xác bằng kết quả STTT. Kết quả được kiểm chứng bằng kết quả giải phẫu bệnh.

Trong nghiên cứu, chúng tôi có 5 trường hợp được chẩn đoán là bướu lành tính trước mổ (tỷ lệ 4,7\%), được thực hiện STTT trong mổ, xác định là UTBMTG. Kết quả nghiên cứu cho thấy việc đánh giá tổn thương trong mổ và kinh nghiệm phẫu thuật viên là rất quan trọng: khi phẫu thuật cho những người bệnh có nguy cơ ung thư thấp (dựa vào khám lâm sàng; siêu âm kích thước u nhỏ, phân nhóm Tirads I,II,III; kết quả FNA lành tính), không nên bỏ qua bước đánh giá đại thể tổn thương một cách tỷ mỹ (ranh giới, bề mặt, xâm lấn...), nểu nghi gờ cần bổ sung xét nghiêm STTT để xác định lại bản chất tổn thương. Mặc dù, việc làm này gẩy kéo dài cuộc mổ nhưng có thể sẽ giúp người bệnh tránh được một cuộc mổ lại để cắt toàn bộ tuyến giáp, vét hạch cổ sau đó.

Một vấn đề cũng cần lưu tâm trong thực hiện FNA và STTT với u tuyến giáp thể nang vì rất khó chẩn đoán lành tính hay ác tính thông qua FNA và STTT, chủ yếu do không có khả năng đánh giá u nang vì mẫu bệnh phẩm không đầy đủ và chẩn đoán không dựa vào các đặc điểm cấu trúc như xâm lấn vỏ như trong carcinoma tuyến giáp thể nhú [6], [7]. Do đó, các nghiên cứu này khuyến cáo không nên STTT đối với các tổn thương dạng nang, cần chờ kết quả giải phẫu bệnh đề quyết định kế hoạch điều trị.

\section{KẾT LUÂ̂N}

Hiệu quả chẩn đoán UTBMTG bằng chọc hút tế bào kim nhỏ đạt $48,1 \%$. Cần chỉ định STTT cho những trường hợp có kết quả FNA nghi ngờ UTBMTG và những trường hợp ghi nhận tổn thương đại thể bất thường trong mổ để nâng cao kết quả chẩn đoán. 
TÀI LIÊU THAM KHẢO

1. Galliano Gretchen $E$, Frishberg David $P$ (2012), "Pathology and classification of thyroid tumors", Thyroid cancer, Springer, 1-33.

2. Lim Raymond ZM, Ooi Juin $\mathbf{Y}$, Tan Jih $\mathrm{H}$, et al. (2019), "Outcome of Cervical Lymph Nodes Dissection for Thyroid Cancer with Nodal Metastases: A Southeast Asian 3-Year Experience", International journal of surgical oncology, 2019.

3. Sung Hyuna, Ferlay Jacques, Siegel Rebecca L, et al. (2021), "Global cancer statistics 2020: GLOBOCAN estimates of incidence and mortality worldwide for 36 cancers in 185 countries", J CA: A Cancer Journal for Clinicians.

4. Crowe Amanda, Linder Ami, Hameed Omar et al. (2011), "The impact of implementation of the Bethesda System for Reporting Thyroid Cytopathology on the quality of reporting, "risk" of malignancy, surgical rate, and rate of frozen sections requested for thyroid lesions", J Cancer cytopathology, 119(5), 315-321.
5. Ye Qin, Woo Jennifer S, Zhao Qunzi, et al. (2017), "Fine-needle aspiration versus frozen section in the evaluation of malignant thyroid nodules in patients with the diagnosis of suspicious for malignancy or malignancy by fine-needle aspiration", ] Archives of pathology laboratory medicine, 141(5), 684-689.

6. LiVolsi Virginia A, Baloch Zubair W (2005), "Use and abuse of frozen section in the diagnosis of follicular thyroid lesions", J Endocrine pathology, 16(4), 285-293.

7. Lumachi Franco, Borsato Simonetta, Tregnaghi Alberto, et al. (2009), "FNA cytology and frozen section examination in patients with follicular lesions of the thyroid gland", J Anticancer research, 29(12), 5255-5257.

8. Huber Gerhard F, Dziegielewski Peter, Matthews T Wayne, et al. (2007), "Intraoperative frozen-section analysis for thyroid nodules: a step toward clarity or confusion?", ] Archives of Otolaryngology-Head Neck Surgery, 133(9), 874-881.

\section{ĐÁNH GIÁ HIÊUU QUẢ PHẪU THUÂT AMIDAN QUÁ PHÁT ĐIỀU TRI NGÁY Ở BÊNNH NHÂN TRƯỞNG THÀNH TAII BÊ̂NH VIÊ̂N TRƯỜNG ĐẠI HỌC Y DƯợC CẦN THO'}

\section{TÓM TẮT}

Mục tiêu: Đánh giá kết guả cải thiên triệu chứng ngáy bŭ̀ng phương pháp phẩu thuật cắt amidan quá phát ở người trưởng thành. Đối tượng và phương pháp: Nghiên cứu tiến cứu có can thiệp lâm sàng trên 49 bệnh nhân từ 18 tuổi trở lên được chẩn đoán ngáy và đươơc điêuu trị bằng phẫu thuât cắt amidan quá phát. Địa điểm tại bệnh viện trường Đại Học Y Dược Cần Thơ và bênh viền Tai Mũi Hong Cần Thơ, thời gian từ tháng 08/2018 đến 02/2021. Kết quả: Có 49 bênh nhân được đưa vào nghiên cứu, trong đó có 38 nam $(63,3 \%), 11$ nữ $(36,7 \%)$. Độ tuối trung bình của đối tượng nghiên cứu là $38,89 \pm 7,8$.Trung bình BMI: 28,5 . Độ ngáy chiếm tỷ lệ cao nhất là độ III $(44,9 \%)$. Amidan quá phát độ III có tỳ lệ cao nhất $(72,22 \%)$. Không có trường hợp nàobi tai biến trong phẫu thuâtt. Tỷ lệ bệnh nhân có kết quả tốt là khá cao, khi ra viện chiếm $(75,5 \%)$ và sau 3 tháng chiếm $(93,8 \%)$. Kết luận: Phấu thuật cắt amiđan quá phát làm rộng eo họng, rộng đường hô hấp và sẽ giúp bệnh nhân cải thiện tình trang ngáy đáng kể.

Tứ khoá: Ngủ ngáy, amidan quá phát, phẫu thuật cắt amidan

\section{*Trường Đại học Y Dược Cần Thơ}

Chịu trách nhiệm chính: Nguyễn Triều Việt

Email: vietctho@gmail.com

Ngày nhận bài: 10/8/2021

Ngày phản biện khoa học: 29/8/2021

Ngày duyệt bài: 24/9/2921
Nguyễn Triều Việt*, Nguyễn Minh Dương*

\section{SUMMARY}

EVALUATING THE RESULT OF TONSILLECTOMY FOR TREATING THE TONSIL HYPERTROPHY CAUSING SNORING IN ADULTS AT CAN THO UNIVERSITY OF MEDICINE AND PHARMACY HOSPITAL

Objectives: Evaluating the results of tonsillectomy in patients with tonsillar hypertrophy to treating snoring. Materials and methods: A descriptive and interventional study was conducted on 49 ADULTswho were diagnosed with snoring and treated with tonsillectomy. Our study was conducted in Can Tho University Hospital of Medicine And Pharmacy from 4/2018 to 4/2020. Results: 49 patients were included in the study (38 males $(63.3 \%)$ and 11 females $(36.7 \%)$. The average age of the study subjects is $38.89 \pm 7.8$. Average BMI: 28.5. Snoring accounted for the highest rate of level III (44.9\%).Grade III hypertrophic tonsillitis has the highest rate $(72.22 \%)$. There in no complication happenning in the operations. The proportion of patients with good results is quite high, at discharge (75.5\%) and after 3 months (93.8\%). Conclusion: Tonsillectomy in patients with tonsillar hypertrophy will widen the waist of the throat, the airways and will help the patient improve the snoring significantly.

Keywords: Snoring, hypertrophic tonsillitis, tonsillectomy

\section{I. ĐẶT VẤN ĐỀ}

Ngáy là biểu hiện đầu tiên và thường gặp của 